\title{
Greedy Heuristic Resource Allocation Algorithm for Device-to-Device Aided Cellular Systems with System Level Simulations
}

\author{
Xianxian Wang ${ }^{1}$, Shaobo $\mathrm{Lv}^{1}$, Xing Wang ${ }^{2}$, and Zhongshan Zhang ${ }^{1}$ \\ ${ }^{1}$ Beijing Engineering and Technology Center for Convergence Networks and Ubiquitous Services, \\ University of Science and Technology Beijing (USTB), \\ Beijing, China 100083 \\ ${ }^{2}$ The third office, Tangjialing Road No.1, Beijing Haidian district, Beijing 100094, China \\ [e-mail: wangxianxian_sky@163.com; lvshaoboftd@163.com; wangxing0515@139.com; \\ zhangzs@ustb.edu.cn] \\ *Corresponding author: Zhongshan Zhang
}

Received July 1, 2017; revised October 29, 2017; accepted November 15, 2017; published April 30, 2018

\begin{abstract}
Resource allocation in device-to-device (D2D) aided cellular systems, in which the proximity users are allowed to communicate directly with each other without relying on the intervention of base stations (BSs), is investigated in this paper. A new uplink resource allocation policy is proposed by exploiting the relationship between D2D-access probability and channel gain among variant devices, such as cellular user equipments (CUEs), D2D user equipments (DUEs) and BSs, etc., under the constraints of their minimum signal to interference-plus-noise ratio (SINR) requirements. Furthermore, the proposed resource-allocation problem can be formulated as the cost function of "maximizing the number of simultaneously activated D2D pairs subject to the SINR constraints at both CUEs and DUEs". Numerical results relying on system-level simulations show that the proposed scheme is capable of substantially improving both the D2D-access probability and the network throughput without sacrificing the performance of conventional CUEs.
\end{abstract}

Keywords: Device-to-Device (D2D); Signal to Interference-plus-Noise Ratio (SINR); D2D-Access Probability; Sum Throughput.

This work was supported by the key project of the National Natural Science Foundation of China (No. 61431001) the open research fund of National Mobile Communications Research Laboratory Southeast University (No.2017D02), Key Laboratory of Cognitive Radio and Information Processing, Ministry of Education (Guilin University of Electronic Technology), and the Foundation of Beijing Engineering and Technology Center for Convergence Networks and Ubiquitous Services. 


\section{Introduction}

$\mathbf{W}_{\text {ith the rapid development of wireless communication techniques as well as the rapid }}$ popularity of smart terminals (e.g. ipad and iphone), the existing cellular networks are becoming increasingly difficult to meet the customers' exponentially growing traffic demands [1]-[3].Therefore, both wireless spectrum efficiency and network capacity need to be substantially enhanced [4]-[6]. Meanwhile, the base stations (BSs) may often operate at an overloaded state due to the existing BS-centric architecture of wireless access networks (WANs), consequently resulting in a serious load imbalance over the whole network.

Device-to-Device (D2D) communication technology, which allows proximity users to exchange data directly without relying on the intervention of BSs [7], has been regarded as one of the effective ways for improving system performance [8]-[10] by addressing the above-mentioned issues mainly based on the following benefits. On the one hand, employing "proximity communication" is capable of offering a higher channel quality for proximity-communication peers, corresponding to attaining a higher channel capacity [11], [12] as well as a lower power consumption [13], [14]. On the other hand, a much lower delay than in conventional cellular communications can be guaranteed by enabling direct transmissions between proximity peers [15]. Furthermore, through reusing the licensed spectrum of conventional cellular user equipments (CUEs), the spectral efficiency of wireless networks can be substantially improved by activating D2D links [16]-[18].

However, activating the D2D links may impose a severe interference on the conventional CUEs, thus significantly eroding the performance of the latter [19]-[21]. To achieve a better overall system performance while guaranteeing the minimum Quality of Service (QoS) requirement of CUEs, an appropriate interference management technique (e.g., in terms of resource allocation, mode selection and power control, etc) must be implemented in the activated D2D user equipments (DUEs).

Up to now, interference-management technologies for D2D aided cellular systems have been widely studied in both academy and industry [22],[23]. To coordinate the interference among CUEs and DUEs, the authors in [24] derived the optimal power allocation for optimizing the sum data rate relying on power control schemes for different modes. Subject to a sum data rate constraint, a distributed power control algorithm relying on small-scale path losses has been proposed in [25] for minimizing the overall power consumption. Furthermore, a resource allocation scheme, in which the local awareness of the interference between CUEs and DUEs can be generated at the BSs, is proposed for minimizing the interference imposed on the CUEs [26]. In order to better exploit the advantages of both resource allocation and power control techniques, a joint resource allocation and power control scheme has been proposed in [27] for maximizing the energy-efficiency (EE) of D2D aided underlaying cellular networks. In addition, a scheme by jointly considering mode selection, channel assignment and power control simultaneously in D2D communications has been proposed in [28] for optimizing the overall system throughput while guaranteeing the minimum signal to interference-plus-noise ratio (SINR) of both CUEs and DUEs.

In this paper, resource allocation in D2D aided cellular systems is investigated. A heuristic resource allocation algorithm is proposed for maximizing the number of simultaneously activated D2D pairs. Unlike [29], in the proposed system model, we assume that there exists an interference limited area (ILA) either for BSs or for D2D receivers, inside 
which the licensed spectrum is prohibited to be reused by DUEs. Furthermore, we investigate the relationship between $\mathrm{D} 2 \mathrm{D}$-access ratio and channel gain among variant devices, such as CUEs, DUEs and BSs. Meanwhile, we use the singleton maximization model to analyze the global maximization under the constrains of both SINR and ILA.

The remainder of this paper is organized as follows. Section 2 gives out the system model. The proposed resource allocation strategy is discussed in Section 3, followed by evaluating the performance of the proposed algorithm using simulations in Section 4. Finally, Section 5 concludes this paper.

\section{System Model}

In this section, system model for the proposed D2D aided cellular networks is analyzed, followed by analyzing the SINR of wireless links. After that, a new framework for resource allocation in the proposed system is given out.

\subsection{System Model for D2D Aided Cellular Networks}

In this paper, without loss of generality, a single cell is considered, and licensed spectrum allocated to CUEs is allowed to be fully reused by D2D pairs, as illustrated in Fig. 1. Meanwhile, multiple D2D pairs are allowed to reuse the licensed spectrum of any individual CUE. However, an orthogonal spectrum is assumed to be allocated to an adjacent cell in order to avoid the inter-cell interference. Furthermore, uplink resource sharing is considered in the proposed system model, in which the D2D-induced interference is mainly imposed on BSs and the geographically close-by D2D receivers.

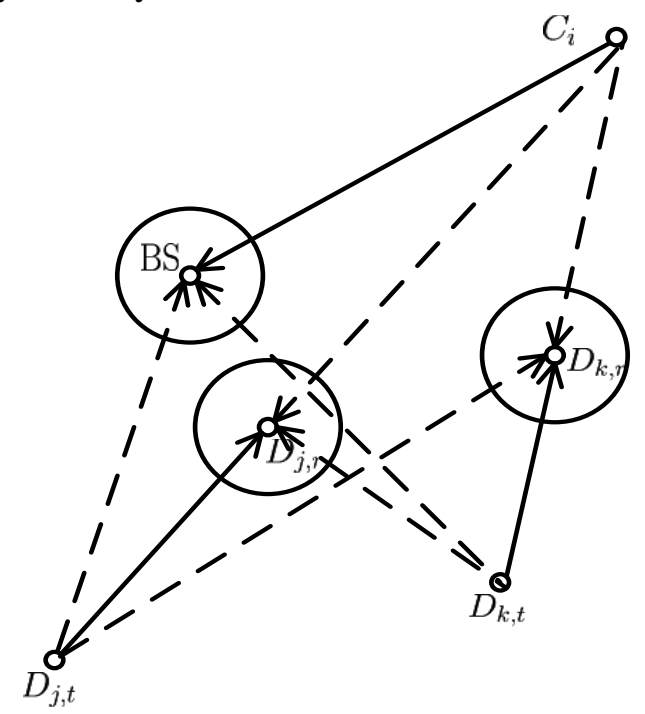

Fig. 1. System model for D2D aided cellular networks, in which multiple D2D pairs are permitted to reuse the licensed spectrum allocated to an individual CUE, where $C_{i}$ and $D_{j, t}\left(D_{k, t}\right), D_{j, r}$ $\left(D_{k, r}\right), \quad i \in\left(1, N_{c}\right), \quad j \in\left(1, N_{d}\right)$, denote CUE and D2D transmitter, D2D receiver, respectively.

A fully-loaded spectrum allocation scenario is considered, in which all the spectrum resources are assumed to be allocated to the CUEs (i.e. there exists no spare spectrum). To mitigate the D2D-induced interference inside a given cellular coverage, a circle guard zone, namely the ILA, is pre-set for both BSs and D2D receivers, inside which area the licensed 
spectrum is prohibited to be reused by D2D transmitters. Before one D2D pair accessing the network, the BS must detect the distance between D2D transimitter and BS. After that, the BS will inform the D2D receivers the objective CUE's location, relying on which the D2D pair can decide whether to reuse the licensed resource or not. In the following, the radius of the ILA is denoted by d. Without loss of generality, all UEs are assumed to be uniformly distributed over the whole cellular coverage. Furthermore, similar to the CUEs, the DUEs also have their own minimum QoS requirements.

\subsection{SINR Analysis for Cellular and D2D links}

In this paper, without loss of generality, a typical Urban Micro (UMi) scenario is considered for modeling the proposed system. The performance erosion is assumed to be induced mainly by the impact of propagation and shadowing effects of wireless channels. Meanwhile, both the antenna gains of devices (i.e. including both BSs and CUEs/DUEs) and the feeder loss are taken into account. For convenience, we use $C=\left\{1,2, \ldots, N_{c}\right\}$ and $D=\left\{1,2, \ldots, N_{d}\right\}$ to denote the index sets of active CUEs and candidate D2D pairs, respectively, with $N_{c}$ and $N_{d}$ denoting the maximum number of CUEs and candidate D2D pairs, respectively. Furthermore, $\phi_{i} \subseteq D$ is used to denote the set of admitted D2D pairs (i.e. the activated DUE pairs), which will reuse the spectrum allocated to the $i$-th CUE. In addition, parameters $C_{i}$ and $D_{j}$ are used to denote the $i$-th CUE and $j$-th D2D pair, respectively.

The received SINR of the typical CUE-BS (i.e., $C_{i}$-BS) and D2D (i.e., $D_{j}$ ) links can be respectively represented as

$$
\left\{\begin{array}{l}
\gamma_{i}^{c}=\frac{P_{0} g_{i, B}}{N_{0}+\sum_{j \in \phi_{i}} P_{0} g_{j, B}}, \\
\gamma_{j}^{d}=\frac{P_{0} g_{j}}{N_{0}+\sum_{k \in \phi_{i} \backslash j} P_{0} g_{k, j}+P_{0} g_{i, j}},
\end{array}\right.
$$

where $\gamma_{i}^{c}$ denotes the SINR of $C_{i}$-BS link, $\gamma_{j}^{d}$ denotes the SINR of D2D $\operatorname{link} D_{j}, P_{0}$ stand for the transmit power of users. Furthermore, $g_{i, B}$ denotes the channel gain between $C_{i}$ and the corresponding BS, and $g_{j, B}$ denotes the channel gain between the $j$-th D2D transmitter and the associated BS. Thus, $g_{j}$ can be used to denote the corresponding channel gain of $D_{j}$, with $g_{i, j}$ standing for the channel gain between $C_{i}$ and the $j$-th D2D receiver. In addition, $\sum_{j \in \phi_{i}} P_{0} g_{j, B}$ is used to represent the interference power imposed on BS by $D_{j}$, and $\sum_{k \in \phi_{i} \backslash j} P_{0} g_{k, j}+P_{0} g_{i, j}$ denotes the sum interference power imposed on $D_{j}$ induced by $C_{i}$ and other D2D pairs. Finally, $N_{0}$ is used to denote the power spectrum density of thermal noise. The sum throughput of the proposed D2D aided cellular systems can thus be expressed as 


$$
R_{\text {sum }}=\sum_{i \in C}\left(\log \left(1+\gamma_{i}^{c}\right)+\sum_{j \in \phi_{i}} \log \left(1+\gamma_{j}^{d}\right)\right) .
$$

\subsection{Formulating the Proposed Resource Allocation Problem}

It has been proven that the system performance in terms of sum throughput can be substantially improved by activating appropriate D2D links [23]. In the system model considering fully spectrum loaded scenario, the licensed spectrum allocated to any individual CUE is allowed to be reused by more than one D2D pair. For convenience of analysis, we can form a frequency reuse set comprising the objective CUE and its co-spectrum DUEs, provided that the minimum QoS (or in other words, minimum SINR) requirement of each user in this set can be guaranteed. Based on the above-mentioned principle, a resource-allocation framework can thus be formulated as:

$$
\begin{array}{ll}
P 1: & \max _{\phi_{1}, \phi_{2}, \ldots, \phi_{N_{c}}} \sum_{i \in C}\left|\phi_{i}\right| \\
\text { s.t. } & \gamma_{i}^{c} \geq \gamma_{\min }^{c}, \forall_{i} \in C, \\
& \gamma_{j}^{d} \geq \gamma_{\min }^{d}, \forall_{j} \in D, \\
& \phi_{p} \cap \phi_{q}=\varnothing, \forall_{p, q} \in C \& p \neq q, \\
& d_{j, B}>d, d_{t, j}>d, \forall_{t} \in C \| D \backslash j, \forall_{j} \in D,
\end{array}
$$

where $\phi_{i}$ represents the set of admitted D2D pairs reusing the spectrum of $C_{i}$, and $\gamma_{\min }^{c}$ and $\gamma_{\min }^{d}$ stand for the minimum SINR requirement of $C_{i}$ and $D_{j}$, respectively. We also use $d_{j, B}$ to denote the distance between $j$-th D2D transmitter and BS, and use $d_{t, j}$ to denote the distance between interference transmitter (i.e. CUE or other D2D transmitter) and $j$-th D2D receiver. Furthermore, (3c) ensures that any D2D pair is permitted to reuse the spectrum of one and only one CUE. In addition, (3d) is used to limit the D2D-induced interference imposed on both BSs and D2D receivers (i.e. corresponding to formulating the function of ILA).

\section{Resource Allocation Strategy For D2D Aided Cellular Systems}

It is shown that the activated D2D links may impose a severe interference on the CUEs [23], and, the interference induced by either CUEs or the geographically close-by D2D transmitters may also significantly erode the quality of a given activated D2D link. To mitigate the D2D-induced interference, an appropriate resource allocation scheme, which is capable of balancing the probability of D2D access and the sum throughput of the whole system, should be implemented.

In this section, a resource allocation strategy aiming at maximizing the number of simultaneously activated D2D pairs while satisfying the minimum QoS requirements of all users (i.e. comprising both CUEs and DUEs) is proposed. According to (3a), and (3b), the maximum number of simultaneously activated D2D pairs can be expressed as 
when $\gamma_{i}^{c} \geq \gamma_{\min }^{c},\left|\phi_{i}\right| \leq \frac{P_{0} g_{i, B}}{\gamma_{\min }^{c} \overline{I_{i}^{c}}}-\frac{N_{0}}{-}$,

when $\gamma_{j}^{d} \geq \gamma_{\min }^{d},\left|\phi_{i}\right| \leq \frac{P_{0} g_{j}}{\gamma_{\min }^{d} I_{j}^{d}}-\frac{N_{0}}{-I_{j}^{d}}$,

thus leading to

$$
\left|\phi_{i}\right| \leq \min \left(\frac{P_{0} g_{i, B}}{\gamma_{\min }^{c} \overline{I_{i}^{c}}}-\frac{N_{0}}{\overline{I_{i}^{c}}}, \frac{P_{0} g_{j}}{\gamma_{\min }^{d} \overline{I_{j}^{d}}}-\frac{N_{0}}{-\overline{I_{j}^{d}}}\right)
$$

where $\overline{I_{i}^{c}}$ and $\overline{I_{j}^{d}}$ denote the average interference $(\bar{I})$ imposed on $C_{i}$ and $D_{j}$ receivers, respectively. In the following, we set $\Phi_{1}=\frac{P_{0} g_{i, B}}{\gamma_{\min }^{c} I_{i}^{c}}-\frac{N_{0}}{-}$ and $\Phi_{2}^{c}=\frac{P_{0} g_{j}}{\gamma_{\min }^{d} I_{j}^{d}}-\frac{N_{0}}{-I_{j}^{d}}$, where $I_{i}^{c}=\sum_{j \in \phi_{i}} P_{0} g_{j, B}$ and $I_{j}^{d}=\sum_{t \in C \| D \backslash j} P_{0} g_{t, j}$, and $\Phi_{1}$ is a monotonically decreasing function of $\overline{I_{i}^{c}}$. Likewise, $\Phi_{2}$ is a monotonically decreasing function of $\overline{I_{j}^{d}}$, when both $\overline{I_{i}^{c}}>0$ and $\overline{I_{j}^{d}}>0$ are satisfied, we have $\overline{I_{i}^{c}}=\overline{I_{j}^{d}}=\bar{I}_{\max }^{-}=P_{0} g_{\max }$ when $g_{j, B}=g_{t, j}=g_{\max }$ (i.e. the interfering transmitter is located at the boundary of the interference limitation area), on the other hand, we have $\Phi_{1} \geq \Phi_{\min }^{1}=\frac{P_{0} g_{i, B}-\gamma_{\min }^{c} N_{0}}{\gamma_{\min }^{c} I_{\max }^{-}}$and $\Phi_{2} \geq \Phi_{\min }^{2}=\frac{P_{0} g_{j}-\gamma_{\min }^{d} N_{0}}{\gamma_{\min }^{d} I_{\max }^{-}}$. Meanwhile, $\left|\phi_{i}\right|$ should satisfy

$$
\left|\phi_{i}\right| \leq \min \left(\Phi_{\min }^{1}, \Phi_{\min }^{2}\right),
$$

Therefore, we can get the maximum- $\left|\phi_{i}\right|$ as

$$
\left|\phi_{\max }^{i}\right|=\left\{\begin{array}{l}
\Phi_{\min }^{1}, \frac{g_{i, B}}{g_{j}} \leq \frac{\gamma_{\min }^{c}}{\gamma_{\min }^{d}}, \\
\Phi_{\min }^{2}, \frac{g_{i, B}}{g_{j}}>\frac{\gamma_{\min }^{c}}{\gamma_{\min }^{d}} .
\end{array}\right.
$$

From (6), we can readily prove that $\frac{g_{i, B}}{g_{j}} \leq \frac{\gamma_{\min }^{c}}{\gamma_{\min }^{d}}$, showing that the maximum of simultaneously activated D2D pairs is $\Phi_{\min }^{1}=\frac{P_{0} g_{i, B}-\gamma_{\min }^{c} N_{0}}{\gamma_{\min }^{c} I_{\max }^{-}}$. Note that the above-mentioned conclusion can be extended to arbitrary $C_{i}, D_{j}$ and 
$\Phi_{i, c}^{1}=\frac{P_{0} g_{i, B}-\gamma_{\min }^{c} N_{0}}{\gamma_{\min }^{c} P_{0} g_{j, B}}$. Thus, to maximize $\Phi_{i, c}^{1}$, we can take $g_{i, B}-\gamma_{\min }^{c} g_{j, B}>\frac{\gamma_{\min }^{c} N_{0}}{P_{0}}$ as a reuse constraint for selecting $D_{j}$. Similarly, if both $\frac{g_{i, B}}{g_{j}}>\frac{\gamma_{\min }^{c}}{\gamma_{\min }^{d}}$ and $\Phi_{i, c}^{2}=\frac{P_{0} g_{j}-\gamma_{\min }^{d} N_{0}}{\gamma_{\min }^{d} P_{0} g_{t, j}}$ are satisfied, we may take $g_{j}-\gamma_{\min }^{d} g_{t, j}>\frac{\gamma_{\min }^{d} N_{0}}{P_{0}}$ as a reuse constrain for selecting $D_{j}$. In summary, to maximize the D2D-access ratio, the reuse restrictions can be expressed as

$$
\left\{\begin{array}{l}
g_{i, B}-\gamma_{\min }^{c} g_{j, B}>\frac{\gamma_{\min }^{c} N_{0}}{P_{0}}, \text { when } \frac{g_{i, B}}{g_{j}} \leq \frac{\gamma_{\min }^{c}}{\gamma_{\min }^{d}}, \\
g_{j}-\gamma_{\min }^{d} g_{t, j}>\frac{\gamma_{\min }^{d} N_{0}}{P_{0}}, \text { when } \frac{g_{i, B}}{g_{j}}>\frac{\gamma_{\min }^{c}}{\gamma_{\min }^{d}} .
\end{array}\right.
$$

From the above-mentioned analysis, the optimal constraints for improving the sum throughput of the proposed D2D aided cellular systems under the criteria (7) of maximizing the activated D2D pairs can be derived as follows.

Obviously, the sum throughput $R_{c}$ in the traditional cellular network (i.e. without considering D2D communications) can be expressed as

$$
R_{c}=\sum_{i \in C}\left[\log \left(1+\xi_{i}^{c}\right)\right]
$$

where

$$
\xi_{i}^{c}=\frac{P_{i}^{c} g_{i, B}}{N_{0}},
$$

As compared to (8), the throughput gain brought about by employing D2D mode can be expressed as

$$
R^{G}=R_{\text {sum }}-R_{c}=\sum_{i \in C}\left(\log \left(\frac{\left(1+\gamma_{i}^{c}\right) \prod_{j \in \phi_{i}}\left(1+\gamma_{j}^{d}\right)}{1+\xi_{i}^{c}}\right)\right)
$$

and

$$
\sum_{i \in C}\left(\log \left(\frac{1+\gamma_{i}^{c}+\sum_{j \in \phi_{i}} \gamma_{j}^{d}}{1+\xi_{i}^{c}}\right)\right) \leq R^{G} \leq \sum_{i \in C}\left(\log \left(\frac{\Upsilon}{1+\xi_{i}^{c}}\right)\right),
$$

where

$$
\begin{aligned}
\Upsilon= & +\left(\gamma_{i}^{c}+\sum_{j \in \phi_{i}} \gamma_{j}^{d}\right)+\frac{C_{m-1}^{1}}{2}\left(\gamma_{i}^{c}+\sum_{j \in \phi_{i}} \gamma_{j}^{d}\right)^{2} \\
& +\ldots+\frac{C_{m-1}^{n}}{n+1}\left(\gamma_{i}^{c}+\sum_{j \in \phi_{i}} \gamma_{j}^{d}\right)^{n+1}+\ldots+\frac{C_{m-1}^{m-1}}{m}\left(\gamma_{i}^{c}+\sum_{j \in \phi_{i}} \gamma_{j}^{d}\right)^{m},
\end{aligned}
$$


with $m$ denoting the size of spectrum reuse set, as derived in Appendix. For conventional cellular systems, as a benchmark, it is easy to derive $R_{\text {sum }}=R_{c}$. However, for D2D aided cellular networks, it is shown that $R_{\text {sum }}>R_{c}$ can be met if $\gamma_{i}^{c}+\sum_{j \in \phi_{i}} \gamma_{j}^{d}>\xi_{i}^{c}$ is satisfied. Furthermore, the performance gain in terms of SINR can be expressed as

$$
\begin{aligned}
\gamma_{i, j}^{G} & =\gamma_{i}^{c}+\sum_{j \in \phi_{i}} \gamma_{j}^{d}-\xi_{i}^{c} \\
& =\sum_{j \in \phi_{i}} \frac{P_{0} g_{j}}{N_{0}+\sum_{t \in C \| D \backslash t} P_{0} g_{t, j}}-\frac{\sum_{j \in \phi_{i}} P_{0} g_{j, B} \cdot P_{0} g_{i, B}}{N_{0}\left(N_{0}+\sum_{j \in \phi_{i}} P_{0} g_{j, B}\right)},
\end{aligned}
$$

Let us set both $\Upsilon_{1}=\sum_{j \in \phi_{i}} \frac{P_{0} g_{j}}{N_{0}+\sum_{t \in C \| D \backslash t} P_{0} g_{t, j}}$ and $\Upsilon_{2}=\frac{\sum_{j \in \phi_{i}} P_{0} g_{j, B} \cdot P_{0} g_{i, B}}{N_{0}\left(N_{0}+\sum_{j \in \phi_{i}} P_{0} g_{j, B}\right)}$, where $\Upsilon_{1}$ is a monotonically decreasing function of $g_{t, j}$ and $\Upsilon_{2}$ is a monotonically decreasing function of $g_{j, B}$, when $g_{t, j}>0$ and $g_{j, B}>0$. Furthermore, let us set both $g_{t, j}=g_{\max }$ and $g_{j, B}=g_{\max }$, thus yielding

$$
\begin{aligned}
\gamma_{i, j}^{G} \geq & \frac{\sum_{j \in \phi_{i}} P_{0} g_{j}}{N_{0}+\sum P_{0} g_{\max }}-\frac{\sum P_{0} g_{\max } \cdot P_{0} g_{i, B}}{N_{0}\left(N_{0}+\sum P_{0} g_{\max }\right)}, \\
& \geq \frac{N_{0} \sum_{j \in \phi_{i}} P_{0} g_{j}-\sum P_{0} g_{\max } \cdot P_{0} g_{i, B}}{N_{0}\left(N_{0}+\sum P_{0} g_{\max }\right)},
\end{aligned}
$$

To make $\gamma_{i, j}^{G}>0$, we may take $N_{0} \sum_{j \in \phi_{i}} P_{0} g_{j}-\sum P_{0} g_{\max } \cdot P_{0} g_{i, B}>0$. Therefore

$$
\frac{g_{i, B}}{g_{j}}<\frac{N_{0}}{P_{0} g_{\max }}
$$

Combine (7) and (15), the reuse restrictions can be expressed as

$$
\left\{\begin{array}{l}
g_{i, B}-\gamma_{\min }^{c} g_{j, B}>\frac{\gamma_{\min }^{c} N_{0}}{P_{0}}, \text { when } \frac{g_{i, B}}{g_{j}} \leq \min \left(\frac{\gamma_{\min }^{c}}{\gamma_{\min }^{d}}, \frac{N_{0}}{P_{0} g_{\max }}\right), \\
g_{j}-\gamma_{\min }^{d} g_{t, j}>\frac{\gamma_{\min }^{d} N_{0}}{P_{0}}, \text { when } \frac{g_{i, B}}{g_{j}} \in\left(\frac{\gamma_{\min }^{c}}{\gamma_{\min }^{d}}, \frac{N_{0}}{P_{0} g_{\max }}\right) \& \&\left(\frac{N_{0}}{P_{0} g_{\max }}>\frac{\gamma_{\min }^{c}}{\gamma_{\min }^{d}}\right) .
\end{array}\right.
$$

Note that the proposed resource-allocation problem can be readily formulated as a Maximum Independent Set Problem (MISP) for each CUE, where the D2D pairs (denoted by $D$ ) and the spectrum reuse relationship among different D2D pairs are denoted by the point 
set $V$ and edge set $E$ of an undirected graph $G=(V, E)$, respectively. Since $\phi_{i}$ denotes a complete graph, while $\phi_{i}$ represents the number of points belonging to the complete graph, our task becomes "finding the $\phi_{i}$ with max- $\phi_{i}$ value in $G$ so as to maximize the number of simultaneously activated D2D pairs associated with each CUE (i.e. that reuse the licensed spectrum allocated to that CUE)". Therefore, (3) can be presented as

$$
\begin{array}{ll}
\text { P2: } & \sum_{i \in C^{N}} \max \left|\phi_{i}\right| \\
\text { s.t. } & g_{i, B}>\gamma_{\min }^{c}\left(\frac{N_{0}}{P_{0}}+g_{j, B}\right) \text {, when } \frac{g_{i, B}}{g_{j}} \leq \min \left(\frac{\gamma_{\min }^{c}}{\gamma_{\min }^{d}}, \frac{N_{0}}{P_{0} g_{\max }}\right), \\
& g_{j}>\gamma_{\min }^{d}\left(\frac{N_{0}}{P_{0}}+g_{t, j}\right), \text { when } \frac{g_{i, B}}{g_{j}} \in\left(\frac{\gamma_{\min }^{c}}{\gamma_{\min }^{d}}, \frac{N_{0}}{P_{0} g_{\max }}\right) \& \&\left(\frac{N_{0}}{P_{0} g_{\max }}>\frac{\gamma_{\min }^{c}}{\gamma_{\min }^{d}}\right), \\
& \phi_{p} \cap \phi_{q}=\varnothing, \forall_{p, q} \in C \& p \neq q, \\
& d_{j, B}>d, d_{t, j}>d, \forall_{t} \in C \| D \backslash j, \forall_{j} \in D,
\end{array}
$$

We use the following Fig. 2 to represent the feasible domain, in which we can perform resource reuse. Note that the size between the points is uncertain, like $\min \left(\frac{\gamma_{\min }^{c}}{\gamma_{\min }^{d}}, \frac{N_{0}}{P_{0} g_{\max }}\right) g_{j}$ and $\frac{\gamma_{\min }^{c} N_{0}}{P_{0}}$, we only list one of the possibilities to prove the existence of the solution where $g_{\text {min }}$ denotes the minimum value under the constraint of cell radius. For arbitrary $C_{i}, D_{j}$, the equation (6) can be expressed as $\left|\phi_{j}^{i}\right|=\left\{\begin{array}{l}\frac{P_{0} g_{i, B}-\gamma_{\min }^{c} N_{0}}{\gamma_{\min }^{c} P_{0} g_{j, B}}, \frac{g_{i, B}}{g_{j}} \leq \frac{\gamma_{\min }^{c}}{\gamma_{\min }^{d}}, \\ \frac{P_{0} g_{j}-\gamma_{\min }^{d} N_{0}}{\gamma_{\min }^{d} P_{0} g_{t, j}}, \frac{g_{i, B}}{g_{j}}>\frac{\gamma_{\min }^{c}}{\gamma_{\min }^{d}} .\end{array}\right.$, mathematically, the max- $\left|\phi_{j}^{i}\right|$ can be expressed as the maximum value of the slope between the point $(x, y)$ and $\left(0, \frac{\gamma_{\min }^{c} N_{0}}{P_{0}}\right)$ ( or $\left(0, \frac{\gamma_{\min }^{d} N_{0}}{P_{0}}\right)$ ) in feasible domain.

Relying on the formulated MISP, a greedy heuristic algorithm is also proposed for solving the proposed resource-allocation problem, as elaborated on in Algorithm 1, in which the complexity is $\mathrm{O}\left(N_{c} N_{d}\right)$. 


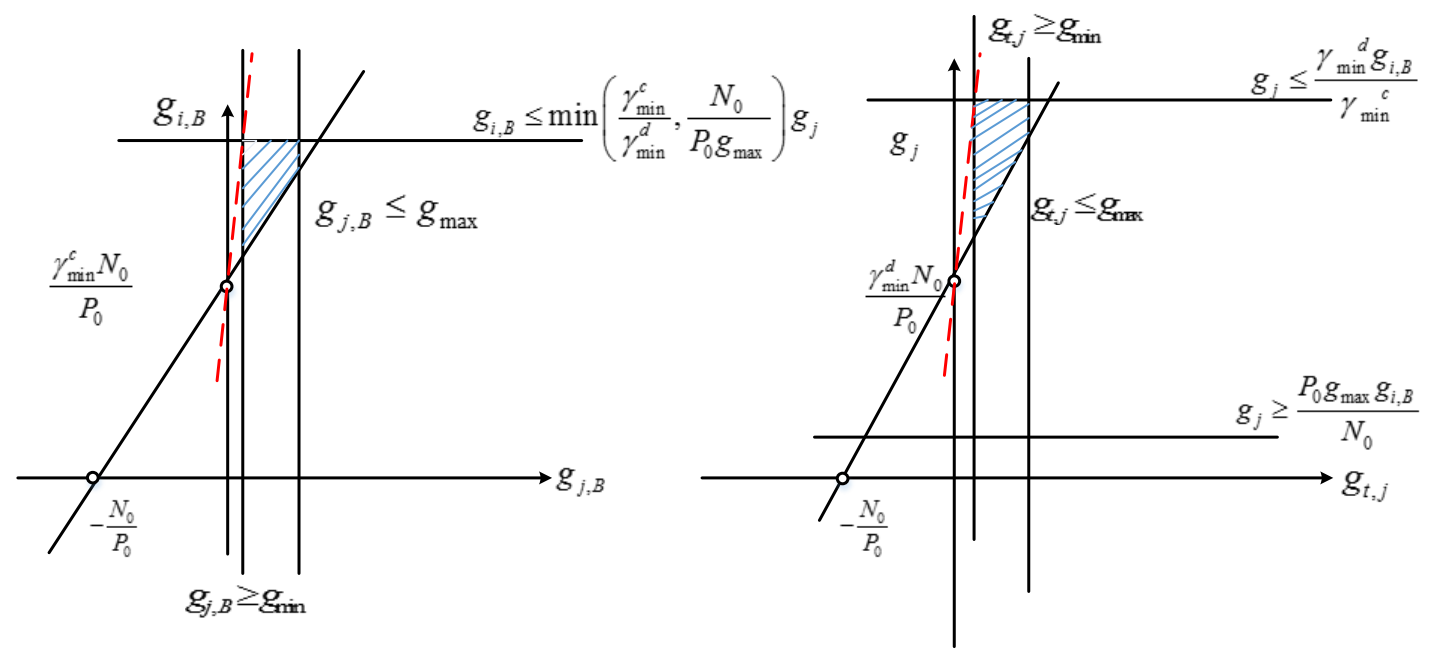

Fig. 2. the possible feasible domain of CUE and DUE

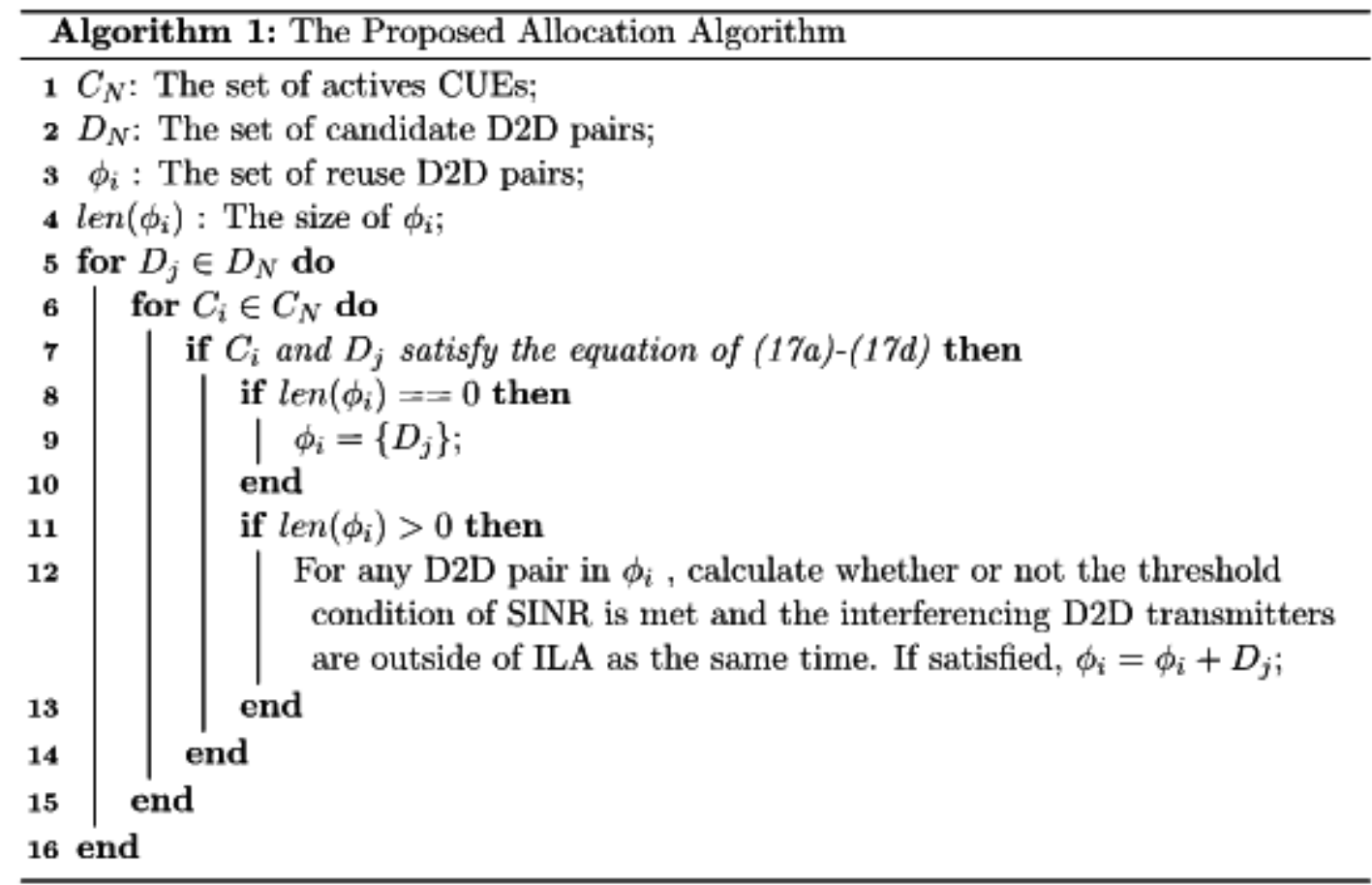

Furthermore, the detailed reuse procedure between D2D pairs and CUE can be expressed as follows:

(1) D2D pairs want to aeccss the network.

(2) The BS chooses the D2D pair according to the session initiation time. If the time is consistent, BS selects the D2D pair according to the distance between D2D peers.

(3) BS selects CUE according to the signal strength of CUE.

(4) BS calculates whether D2D pair and CUE satisfy equations (17a)-(17d). 
(5) If equations (17a)-(17d) are satisfied, BS will send UL grant to D2D pair to start session, followed by picking other D2D pairs, and then repeat (1)-(5).

(6) otherwise, repeat (1)-(5).

\section{Numerical Analysis}

In this section, numerical analysis relying on the proposed channel model is performed for exploring the attainable benefits brought about by employing the proposed algorithm. The objective functions of the proposed optimization is set to be the D2D access ratio (i.e. represented as the number of simultaneously activated D2D pairs to the total number of users), the sum throughput gain over the conventional cellular systems, and D2D link's average throughput parameterized by the increase of CUE-SINR threshold $\eta=\gamma_{\min }^{c}$ and ILA radius $d$.

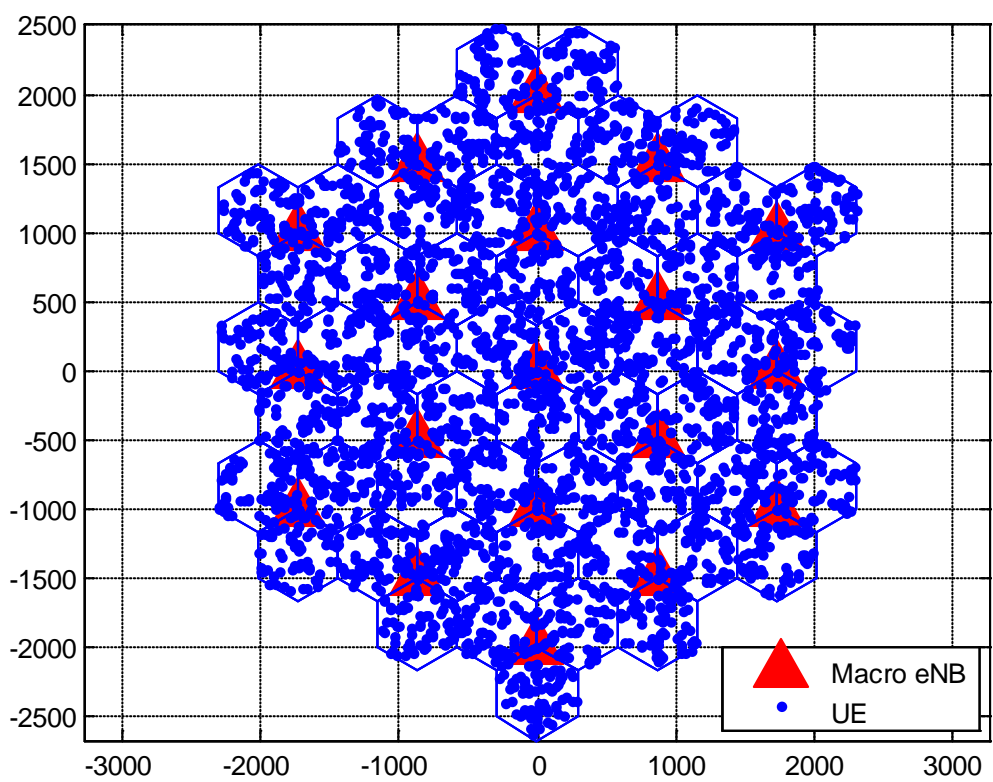

Fig. 3. The simulation is a wrap-around configuration of 19 sites, each comprising 3 cells, inside which the users are distributed uniformly over the whole area.

In the following, similar to [30], a wrap-around system configuration comprising 19 sites (i.e. each comprising 3 cells), with inter-site distance of $1000 \mathrm{~m}$, is considered, as depicted in Fig. 3. Without loss of generality, the minimum UE-to-BS distance is assumed to be $10 \mathrm{~m}$, and the maximum distance between a pair of D2D peers is $50 \mathrm{~m}$. Furthermore, orthogonal spectrum allocation among CUEs is performed. In addition, the transmit power of BS and CUEs assumed to be $42 \mathrm{dBm}$ and $24 \mathrm{dBm}$, respectively. Finally, the noise power spectrum density is assumed to be $-174 \mathrm{dBm} / \mathrm{Hz}$. The detailed parameter settings, which come from the standard ITU-R M.2135[30], are elaborated on in Table 1. 
Xianxian Wang et al.: Greedy Heuristic Resource Allocation Algorithm for Device-to-Device Aided Cellular Systems with System Level Simulations

Table 1. Parameter settings of the proposed system-level simulation.

\begin{tabular}{|c|c|}
\hline Parameters & Settings \\
\hline scenario environment & $\mathrm{UMi}$ \\
\hline system bandwidth & 10MHz (TDD) \\
\hline carrier frequency & $2.5 \mathrm{GHz}$ \\
\hline Inter-site distance (ISD) & $1000 \mathrm{~m}$ \\
\hline the deployment of Macro BS & $\begin{array}{l}\text { typical system layout of } 19 \text { sites, each } \\
\text { comprising } 3 \text { cells with six resolvable } \\
\text { angles }\end{array}$ \\
\hline the min-distance of UE to BS & $10 \mathrm{~m}$ \\
\hline Antenna model of Macro BS & $\begin{array}{l}A(\theta)=-\min \left[12\left(\frac{\theta}{\theta_{3}}\right)^{2}, A_{m}\right] \\
\begin{aligned} & A_{e}(\phi)=-\min \left(12\left(\frac{\phi-\phi_{\text {tilt }}}{\phi_{3}}\right)^{2}, A_{m}\right) \\
&=-\min \left\{-\left(A(\theta)+A_{\varepsilon}(\phi)\right), A_{m}\right\} \\
& A_{m}=20 d B, \quad \theta_{3}=70, \quad \phi_{\text {tilt }}=15 \\
&-180 \leq \theta \leq 180, \quad-90 \leq \phi \leq 90\end{aligned}\end{array}$ \\
\hline Antenna model of UE & Omnidirectional \\
\hline traffic pattern & full buffer \\
\hline BS height & $10 \mathrm{~m}$ \\
\hline UE height & $1.5 \mathrm{~m}$ \\
\hline BS Noise Figure & $5 \mathrm{~dB}$ \\
\hline UE Noise Figure & $7 \mathrm{~dB}$ \\
\hline BS transmit power & $42 \mathrm{dBm}$ \\
\hline UE transmit power & $24 \mathrm{dBm}$ \\
\hline Power level of thermal noise & $-174 \mathrm{dBm} / \mathrm{Hz}$ \\
\hline
\end{tabular}

\subsection{Channel Model for System-Level Simulations}

The Spatial Channel Model (SCM), which is a typical double directional geometry-based stochastic model [30], is adopted in this section. The path loss model of BS-to-UE links is given by ITU-R M.2135 standard, whereas the path loss model of UE-to-UE links has been defined in 3rd Generation Partnership Project (3GPP) specification 36.843-c01[31][32]. Furthermore, two propagation scenarios, including Line of Sight (LOS) and Non Line Of Sight (NLOS) for outdoor users in UMi, are considered. Note that the LOS probability can be parameterized as a function of distance $\mathrm{l}$ :

$$
P_{\text {LOS }}=\min \left(\frac{18}{l}\right)\left(1-\exp \left(\frac{-l}{36}\right)\right)+\exp \left(\frac{-l}{36}\right) \text {. }
$$

Similarly, the UE-to-UE path loss can be expressed as

$$
P_{L}=\max \left(P_{L, \text { free }}, P_{L, b}\right) \text {, }
$$

and 


$$
\left\{\begin{array}{l}
P_{L, \text { free }}=20.0 \log (l)+46.4+20.0 \log \left(f_{c}\right) \text {, if free-space, } \\
P_{L, b}=22.7 \log (l)+27.0+20.0 \log \left(f_{c}\right), \text { if } L O S \& \quad l \in\left(10,4.0 h_{b s} h_{m s} f_{c}\right), \\
P_{L, b}=40.0 \log (l)+7.56-17.3 \log \left(h_{b s}\right)-17.3 \log \left(h_{m s}\right)+2.7 \log \left(f_{c}\right), \\
\text { if } \quad L O S \& \quad l \in\left((0,10),\left(4.0 h_{b s} h_{m s} f_{c}, \infty\right)\right), \\
P_{L, b}=\left(44.9-6.55 \log \left(h_{B S}\right)\right) \log (l)+18.38+5.83 \log \left(h_{B S}\right)+23.0 \log \left(f_{c}\right), \\
\text { if } \quad N L O S,
\end{array}\right.
$$

where $l$ denotes the average distance between a pair of D2D peers, $f_{c}$ stands for the center frequency, $h_{B S}$ and $h_{M S}$ represent the actual antenna height and the effective environmental height, respectively. Furthermore, we have $h_{b s}=h_{B S}-1.0$ and $h_{m s}=h_{M S}-1.0$.

Apart from it, shadowing effect is assumed to follow a log-normal distribution, as expressed as

$$
\delta_{S F, n}=10^{\frac{\gamma_{n} \delta_{S H}}{10}},
$$

where $\gamma_{n}$ is a Gaussian random variable, and $\delta_{S H}$ denotes the shadow fading standard deviation in $\mathrm{dB}$. Meanwhile, the antenna model is given by Table 1 . The channel gain can thus be expressed as

$$
g_{i, j}=\frac{1.0}{\left(P_{L}-S_{F}-A_{i}-A_{j}+\text { feederloss }\right)},
$$

where $P_{L}$ denotes the path loss exponential, $S_{F}$ represents the shadowing effect, $A_{i}$ and $A_{j}$ are the antenna gains of signal transmitter and signal receiver, respectively, and feederloss is set to be $20 \mathrm{~dB}$.

\subsection{Simulation Results}

In this subsection, the performance comparison between the greedy resource allocation algorithm (GRA) and without the greedy resource allocation algorithm in terms of D2D access probability is performed, where the throughput gain of the latter is evaluated subject to the SINR constraints of both CUEs and DUEs.

In Fig. 4, the performance of GRA and without GRA in terms of the maximum number of simultaneously activated D2D pairs is performed by considering variant $\eta$ values, with a single CUE considered. It is shown that the number of simultaneously activated D2D pairs decreases as $\eta$ increases, because the interference tolerance of CUE decreases as the SINR threshold increases, thus requiring fewer D2D pairs to be activated simultaneously so as to impose a lower interference on CUE (i.e. to guarantee the minimum SINR requirement of the CUE). Anyway, the proposed algorithm is shown to always outperform the without GRA in terms of the maximum number of simultaneously activated D2D pairs, because the former is capable of coordinating the interference between CUEs and D2D pairs and optimizing the spectrum reuse set adaptively according to the instantaneous channel condition.

In Fig. 5, the impacts of ILA radius and CUE-SINR threshold on the number of simultaneously activated D2D pairs in a single-cellular scenario is investigated. It is shown 
that the access probability of the proposed algorithm is a monotonically decreasing function of $\eta$, because the interference tolerance of DUEs decreases as $\eta$ increases. Meanwhile, it is also shown that the performance of GRA increases first, and then decreases as $d$ increases. We can explain this observation as follows: when ILA radius is small, the interference imposed on signal receiver from one interference transmitter is more intensive compared with signal receiver power when the distance of interference link is smaller than D2D links. But when ILA radius is beyond the distance of D2D links, the probability of candidate D2D pairs access to net-work declines with the increase of $d$, as a result, the number of admitted D2D pairs decreases.

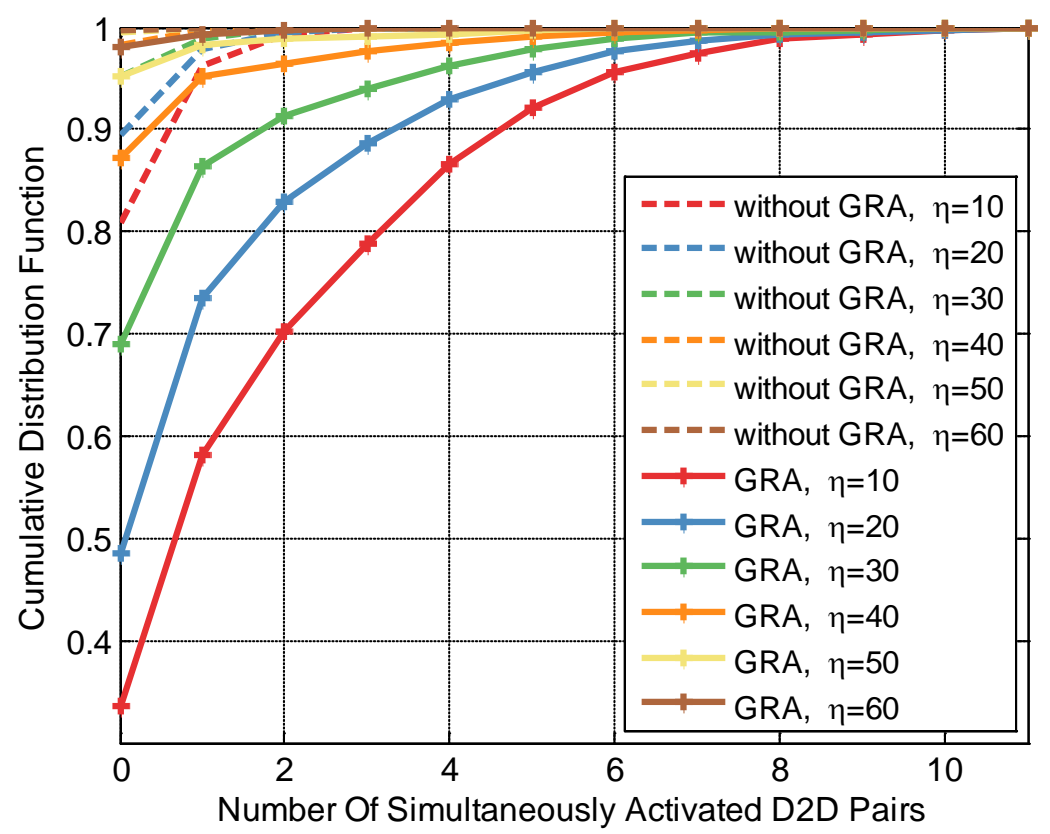

Fig. 4. Performance comparison of the greedy allocation algorithm and the without greedy allocation scheme in terms of the maximum number of simultaneously activated D2D pairs for variant SINR thresholds, with a single CUE considered, where $N_{c}=50, N_{d}=300$ and $d=50$.

Fig. 6 demonstrates the impact of ILA on the throughput as a function of $\eta$. Evidently, the throughput is a monotonically decreasing function of $\eta$, because the number of simultaneously activated D2D pairs decreases as the SINR threshold increases. Meanwhile, the performance of the GRA is also shown to increase firstly, and then decreases as $d$ increases. We can explain it as follows: Since the interference is much more intensive when $d<50$ (i.e. the maximum allowable distance of D2D links) compared with receiver signal power, fewer D2D pairs can access the network under the requirement of "guaranteeing the required ILA radius, if it is beyond the distance of D2D links". Consequently, the sum throughput decreases.

In Fig. 7, the impact of CUE-SINR threshold as a function of $d$ on the D2D throughput is evaluated. It is shown that the performance is a concave function of $d$. We can explain this observation as follows: Increasing the ILA radius implies increasing the distance between the interference transmitters and signal receivers, and the interference imposed on D2D receivers by CUEs as well as that imposed on CUEs by D2D pairs will both decrease. 
However, setting a larger inter-user distance will always reduce the access probability of DUEs. Consequently, as ILA radius increases, the relatively lower interference level will result in an increase in sum throughput in the fist place, and then the lower access probability of DUEs will lead to a reduction in the sum throughput. It is also shown that the performance of the proposed algorithm declines as $\eta$ increases, making this degradation rate become slower as $\eta$ becomes larger, because a higher SINR threshold corresponds to a lower interference tolerance.

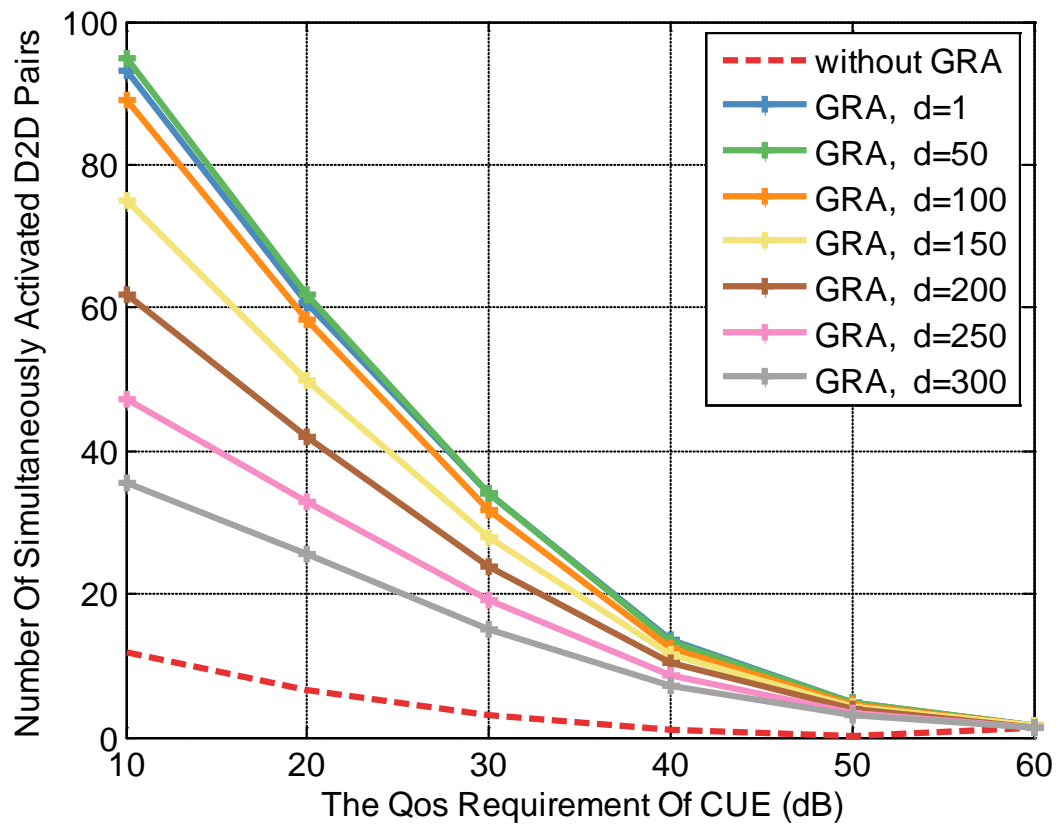

Fig. 5. The maximum number of simultaneously activated D2D pairs in the proposed greedy allocation algorithm system under different settings of SINR threshold, with a BS, where $N_{c}=50$;

$$
N_{d}=300 .
$$

\section{Conclusion}

In this paper, the problem of adaptive spectrum allocation was formulated as the maximization of the number of simultaneously activated D2D pairs in scenario of D2D reusing the uplink licensed spectrum, with a fully loaded cellular system considered. To maximize the number of simultaneously activated D2D pairs without eroding the SINRs of both CUEs and DUEs, a greedy heuristic algorithm was also implemented for finding the objective spectrum-reuse set. Numerical results showed that the proposed algorithm is capable of improving both the D2D-access probability and the sum throughput of the whole system. It was shown that the proposed greedy resource allocation algorithm is capable of improving the D2D-access probability by about $650 \%$ and the sum throughput by about $80 \%$ as compared to the without GRA when $\eta=20 d B$ and $d=50 \mathrm{~m}$. 


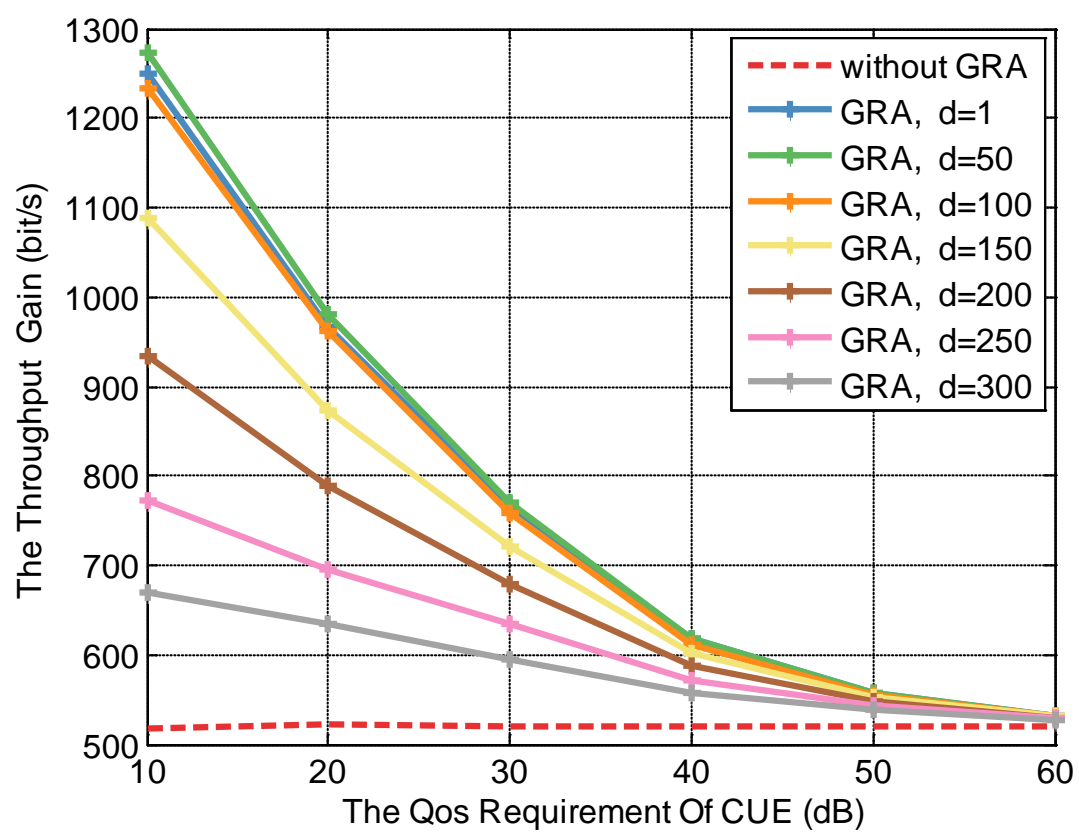

Fig. 6. Performance comparison of the greedy allocation algorithm and the without greedy allocation scheme in terms of the sum throughput gain for variant radius of ILA, where $N_{c}=50$ and $N_{d}=300$.

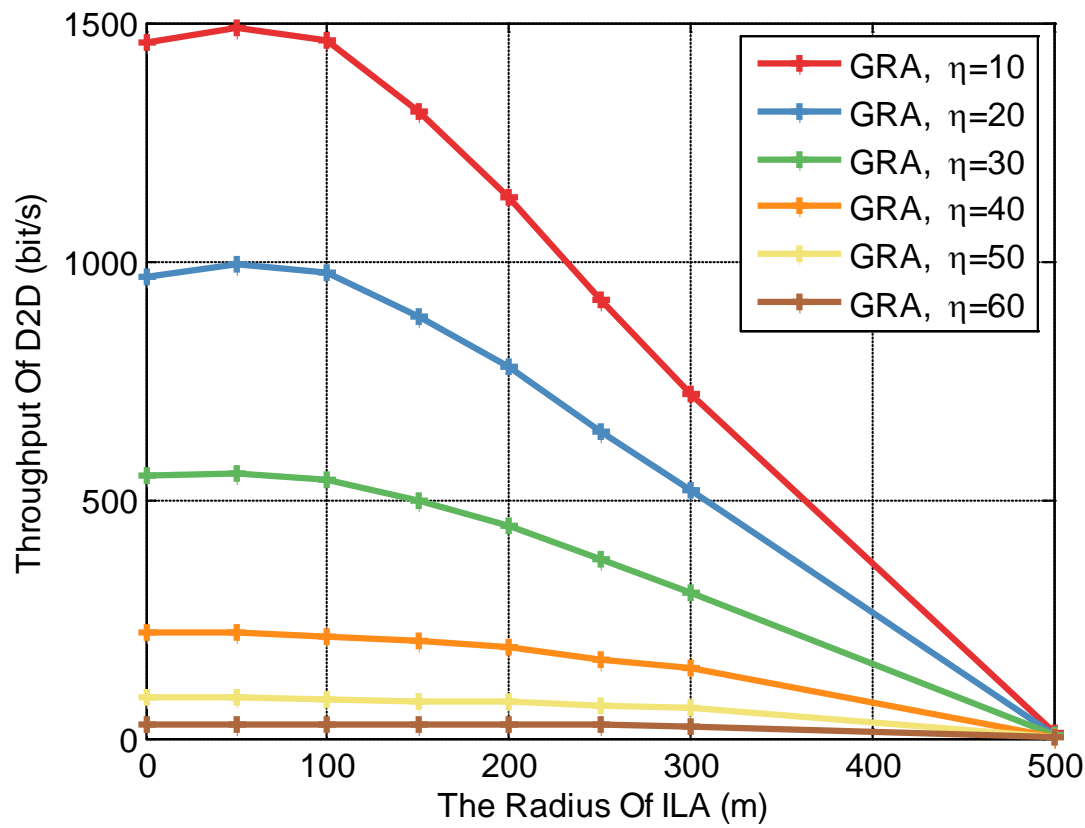

Fig. 7. Sum throughput of D2D links under variant settings of CUE-SINR threshold, where $N_{c}=50$ and $N_{d}=300$. 


\section{Appendix}

The sum throughput of the proposed D2D aided cellular systems can be expressed as

$$
\begin{aligned}
R_{\text {sum }} & =\sum_{i \in C}\left(\log \left(1+\gamma_{i}^{c}\right)+\sum_{j \in \phi_{i}} \log \left(1+\gamma_{j}^{d}\right)\right) \\
& =\sum_{i \in C}\left(\log \left(\left(1+\gamma_{i}^{c}\right) \prod_{j \in \phi_{i}}\left(1+\gamma_{j}^{d}\right)\right)\right),
\end{aligned}
$$

where

$$
\gamma_{i, j}=\left(1+\gamma_{i}^{c}\right) \prod_{j \in \phi_{i}}\left(1+\gamma_{j}^{d}\right),
$$

By further analyzing (25), we have

$$
\begin{aligned}
\gamma_{i, j} & =1+\gamma_{i}^{c}+\sum_{j \in \phi_{i}} \gamma_{j}^{d}+\sum_{j \in \phi_{i}} \gamma_{i}^{c} \gamma_{j}^{d}+\sum_{k \in \phi_{i} \backslash j} \sum_{j \in \phi_{i}} \gamma_{k}^{d} \gamma_{j}^{d}+\ldots+\gamma_{i}^{c} \prod_{j \in \phi_{i}} \gamma_{j}^{d} \\
& \geq 1+\gamma_{i}^{c}+\sum_{j \in \phi_{i}} \gamma_{j}^{d}
\end{aligned}
$$

And, we let $m$ denote the size of reuse set, when $\mathrm{m}=2$, we can get

$$
\begin{aligned}
\gamma_{i, j} & =1+\gamma_{i}^{c}+\gamma_{j}^{d}+\gamma_{i}^{c} \gamma_{j}^{d} \\
& \leq 1+\gamma_{i}^{c}+\gamma_{j}^{d}+\frac{1}{2}\left(\left(\gamma_{i}^{c}\right)^{2}+\left(\gamma_{j}^{d}\right)^{2}\right), \\
& \leq 1+\gamma_{i}^{c}+\gamma_{j}^{d}+\frac{1}{2}\left(\gamma_{i}^{c}+\gamma_{j}^{d}\right)^{2},
\end{aligned}
$$

When $\mathrm{m}=3$, we can get

$$
\begin{aligned}
\gamma_{i, j} \leq & 1+\gamma_{i}^{c}+\gamma_{j_{1}}^{d}+\gamma_{j_{2}}^{d}+\gamma_{i}^{c} \gamma_{j_{1}}^{d}+\gamma_{i}^{c} \gamma_{j_{2}}^{d}+\gamma_{j_{1}}^{d} \gamma_{j_{2}}^{d}+\gamma_{i}^{c} \gamma_{j_{1}}^{d} \gamma_{j_{2}}^{d} \\
\leq & 1+\gamma_{i}^{c}+\sum_{k=1}^{2} \gamma_{j_{k}}^{d}+\frac{1}{2}\left(\left(\gamma_{i}^{c}\right)^{2}+\left(\gamma_{j_{1}}^{d}\right)^{2}\right)+\frac{1}{2}\left(\left(\gamma_{i}^{c}\right)^{2}+\left(\gamma_{j_{2}}^{d}\right)^{2}\right)+\frac{1}{2}\left(\left(\gamma_{j_{1}}^{d}\right)^{2}+\left(\gamma_{j_{2}}^{d}\right)^{2}\right) \\
& +\frac{1}{3}\left(\left(\gamma_{i}^{c}\right)^{3}+\left(\gamma_{j_{1}}^{d}\right)^{3}+\left(\gamma_{j_{2}}^{d}\right)^{3}\right) \\
\leq & 1+\gamma_{i}^{c}+\sum_{k=1}^{2} \gamma_{j_{k}}^{d}+\frac{C_{2}^{1}}{2}\left(\left(\gamma_{i}^{c}\right)^{2}+\left(\gamma_{j_{1}}^{d}\right)^{2}+\left(\gamma_{j_{2}}^{d}\right)^{2}\right)+\frac{C_{2}^{2}}{3}\left(\left(\gamma_{i}^{c}\right)^{3}+\left(\gamma_{j_{1}}^{d}\right)^{3}+\left(\gamma_{j_{2}}^{d}\right)^{3}\right) \\
\leq & 1+\gamma_{i}^{c}+\sum_{k=1}^{2} \gamma_{j_{k}}^{d}+\frac{C_{2}^{1}}{2}\left(\gamma_{i}^{c}+\gamma_{j_{1}}^{d}+\gamma_{j_{2}}^{d}\right)^{2}+\frac{C_{2}^{2}}{3}\left(\gamma_{i}^{c}+\gamma_{j_{1}}^{d}+\gamma_{j_{2}}^{d}\right)^{3},
\end{aligned}
$$

when $m=4$, we can get 


$$
\begin{aligned}
\gamma_{i, j}= & 1+\gamma_{i}^{c}+\gamma_{j_{1}}^{d}+\gamma_{j_{2}}^{d}+\gamma_{j_{3}}^{d}+\gamma_{i}^{c} \gamma_{j_{1}}^{d}+\gamma_{i}^{c} \gamma_{j_{2}}^{d}+\gamma_{j_{1}}^{d} \gamma_{j_{3}}^{d}+\gamma_{j_{1}}^{d} \gamma_{j_{2}}^{d}+\gamma_{j_{1}}^{d} \gamma_{j_{3}}^{d}+\gamma_{j_{2}}^{d} \gamma_{j_{3}}^{d} \\
& +\gamma_{i}^{c} \gamma_{j_{1}}^{d} \gamma_{j_{2}}^{d}+\gamma_{i}^{c} \gamma_{j_{1}}^{d} \gamma_{j_{3}}^{d}+\gamma_{i}^{c} \gamma_{j_{2}}^{d} \gamma_{j_{3}}^{d}+\gamma_{j_{1}}^{d} \gamma_{j_{2}}^{d} \gamma_{j_{3}}^{d}+\gamma_{i}^{c} \gamma_{j_{1}}^{d} \gamma_{j_{2}}^{d} \gamma_{j_{3}}^{d} \\
\leq & 1+\gamma_{i}^{c}+\sum_{k=1}^{3} \gamma_{j_{k}}^{d}+\frac{1}{2}\left(\left(\gamma_{i}^{c}\right)^{2}+\left(\gamma_{j_{1}}^{d}\right)^{2}\right)+\frac{1}{2}\left(\left(\gamma_{i}^{c}\right)^{2}+\left(\gamma_{j_{2}}^{d}\right)^{2}\right) \\
& +\frac{1}{2}\left(\left(\gamma_{i}^{c}\right)^{2}+\left(\gamma_{j_{3}}^{d}\right)^{2}\right)+\frac{1}{2}\left(\left(\gamma_{j_{1}}^{d}\right)^{2}+\left(\gamma_{j_{2}}^{d}\right)^{2}\right)+\frac{1}{2}\left(\left(\gamma_{j_{1}}^{d}\right)^{2}+\left(\gamma_{j_{3}}^{d}\right)^{2}\right) \\
& +\frac{1}{2}\left(\left(\gamma_{j_{2}}^{d}\right)^{2}+\left(\gamma_{j_{3}}^{d}\right)^{2}\right)+\frac{1}{3}\left(\left(\gamma_{i}^{c}\right)^{3}+\left(\gamma_{j_{1}}^{d}\right)^{3}+\left(\gamma_{j_{2}}^{d}\right)^{3}\right)+\frac{1}{3}\left(\left(\gamma_{i}^{c}\right)^{3}+\left(\gamma_{j_{1}}^{d}\right)^{3}+\left(\gamma_{j_{3}}^{d}\right)^{3}\right) \\
& +\frac{1}{3}\left(\left(\gamma_{i}^{c}\right)^{3}+\left(\gamma_{j_{2}}^{d}\right)^{3}+\left(\gamma_{j_{3}}^{d}\right)^{3}\right)+\frac{1}{3}\left(\left(\gamma_{j_{1}}^{d}\right)^{3}+\left(\gamma_{j_{2}}^{d}\right)^{3}+\left(\gamma_{j_{3}}^{d}\right)^{3}\right) \\
& +\frac{1}{4}\left(\left(\gamma_{i}^{c}\right)^{4}+\left(\gamma_{j_{1}}^{d}\right)^{4}+\left(\gamma_{j_{2}}^{d}\right)^{4}+\left(\gamma_{j_{3}}^{d}\right)^{4}\right) \\
\leq & 1+\gamma_{i}^{c}+\sum_{k=1}^{3} \gamma_{j_{k}}^{d}+\frac{C_{3}^{1}}{2}\left(\left(\gamma_{i}^{c}\right)^{2}+\left(\gamma_{j_{1}}^{d}\right)^{2}+\left(\gamma_{j_{2}}^{d}\right)^{2}+\left(\gamma_{j_{3}}^{d}\right)^{2}\right) \\
& +\frac{C_{3}^{2}}{3}\left(\left(\gamma_{i}^{c}\right)^{3}+\left(\gamma_{j_{1}}^{d}\right)^{3}+\left(\gamma_{j_{2}}^{d}\right)^{3}+\left(\gamma_{j_{3}}^{d}\right)^{3}\right)+\frac{C_{3}^{3}}{4}\left(\left(\gamma_{i}^{c}\right)^{4}+\left(\gamma_{j_{1}}^{d}\right)^{4}+\left(\gamma_{j_{2}}^{d}\right)^{4}+\left(\gamma_{j_{3}}^{d}\right)^{4}\right) \\
\leq & 1+\gamma_{i}^{c}+\sum_{k=1}^{3} \gamma_{j_{k}}^{d}+\frac{C_{3}^{1}}{2}\left(\gamma_{i}^{c}+\gamma_{j_{1}}^{d}+\gamma_{j_{2}}^{d}+\gamma_{j_{3}}^{d}\right)^{2} \\
& +\frac{C_{3}^{2}}{3}\left(\gamma_{i}^{c}+\gamma_{j_{1}}^{d}+\gamma_{j_{2}}^{d}+\gamma_{j_{3}}^{d}\right)^{3}+\frac{C_{3}^{3}}{4}\left(\gamma_{i}^{c}+\gamma_{j_{1}}^{d}+\gamma_{j_{2}}^{d}+\gamma_{j_{3}}^{d}\right)^{4},
\end{aligned}
$$

Thus, the generic formula can be expressed as

$$
\begin{aligned}
& \gamma_{i, j}=1+\gamma_{i}^{c}+\sum_{j \in \phi_{i}} \gamma_{j}^{d}+\sum_{j \in \phi_{i}} \gamma_{i}^{c} \gamma_{j}^{d}+\sum_{k \in \phi_{i} \backslash j} \sum_{j \in \phi_{i}} \gamma_{k}^{d} \gamma_{j}^{d} \\
& +\ldots+\gamma_{i}^{c} \underbrace{\sum_{n_{1} \in \phi \backslash n_{2 \ldots n}} \sum_{n_{2} \in \phi \backslash n_{1,3 . n}} \ldots \sum_{n_{n} \in \phi \backslash n_{1 . \ldots n-1}} \gamma_{n_{1}}^{d} \gamma_{n_{2}}^{d} \ldots \gamma_{n_{n}}^{d}}_{n}+\ldots+\gamma_{i}^{c} \prod_{j \in \phi_{i}} \gamma_{j}^{d} \\
& \leq 1+\gamma_{i}^{c}+\sum_{j \in \phi_{i}} \gamma_{j}^{d}+\frac{C_{m-1}^{2-1}}{2}\left(\left(\gamma_{i}^{c}\right)^{2}+\sum_{j \in \phi_{i}}\left(\gamma_{j}^{d}\right)^{2}\right) \\
& +\ldots+\frac{C_{m-1}^{n}}{n+1}\left(\left(\gamma_{i}^{c}\right)^{n+1}+\sum_{j \in \phi_{i}}\left(\gamma_{j}^{d}\right)^{n+1}\right)+\ldots+\frac{C_{m-1}^{m-1}}{m}\left(\left(\gamma_{i}^{c}\right)^{m}+\sum_{j \in \phi_{i}}\left(\gamma_{j}^{d}\right)^{m}\right) \\
& \leq 1+\left(\gamma_{i}^{c}+\sum_{j \in \phi_{i}} \gamma_{j}^{d}\right)+\frac{C_{m-1}^{1}}{2}\left(\gamma_{i}^{c}+\sum_{j \in \phi_{i}} \gamma_{j}^{d}\right)^{2}+\ldots+\frac{C_{m-1}^{n}}{n+1}\left(\gamma_{i}^{c}+\sum_{j \in \phi_{i}} \gamma_{j}^{d}\right)^{n+1} \\
& +\ldots+\frac{C_{m-1}^{m-1}}{m}\left(\gamma_{i}^{c}+\sum_{j \in \phi_{i}} \gamma_{j}^{d}\right)^{m}
\end{aligned}
$$

thus proved (10) and (11). 


\section{References}

[1] Z. Zhang, K. Long, J. Wang, and F. Dressler, “On swarm intelligence inspired self-organized networking: its bionic mechanisms, designing principles and optimization approaches,” IEEE Commun. Surveys Tuts., vol. 16, no. 1, pp. 513-537, FIRST QUARTER 2014. Article (CrossRef Link)

[2] Z. Zhang, K. Long, and J. Wang, "Self-organization paradigms and optimization approaches for cognitive radio technologies: a survey,” IEEE Wireless Commun. Mag., vol. 20, no. 2, pp. 36-42, Apr. 2013. Article (CrossRef Link)

[3] E. Dahlman, S. Parkvall, and J. Skold, “4G: LTE/LTE-advanced for mobile broadband,” Academic press, 2013. Article (CrossRef Link)

[4] Z. Zhang, X. Chai, K. Long, A. V. Vasilakos, and L. Hanzo, "Full duplex techniques for 5G networks: self-interference cancellation, protocol design, and relay selection,” IEEE Commun. Mag., vol. 53, no. 5, pp.128-137, May. 2015. Article (CrossRef Link)

[5] Z. Zhang, K. Long, A. V. Vasilakos, and L. Hanzo, "Full-duplex wireless communications: challenges, solutions and future research directions," in Proc. of IEEE, vol. 104, no. 7, pp. 1369-1409, July. 2016. Article (CrossRef Link)

[6] G. Wang, Q. Liu, R. He, F. Gao, and C. Tellambura, "Acquisition of channel state information in heterogeneous cloud radio access networks: challenges and research directions," IEEE Wireless Communications, vol. 22, no. 3, pp. 100-107, June. 2015. Article (CrossRef Link)

[7] S.-Y. Lien, C.-C. Chien, F.-M. Tseng, and T.-C. Ho, "3gpp deviceto-device communications for beyond 4g cellular networks,” IEEE Communications Magazine, vol. 54, no. 3, pp. 29-35, Mar. 2016. Article (CrossRef Link)

[8] H. Xiao and S. Ouyang, "Power allocation for a hybrid decode-amplify-forward cooperative communication system with two source-destination pairs under outage probability constraint," IEEE Systems Journal, vol. 9, no. 3, pp. 797-804, Sept. 2015. Article (CrossRef Link)

[9] G. Wang, F. Gao, and C. Tellambura, "Ambient backscatter communication systems: detection and performance analysis,” IEEE Trans.Commun., vol. 64, no. 11, pp. 4836-4846, Nov. 2016. Article (CrossRef Link)

[10] C. Xing, S. Ma, Z. Fei, Y.-C. Wu, and H. V. Poor, “A general robust linear transceiver design for multi-hop amplify-and-forward mimo relaying systems," IEEE Transactions on Signal Processing, vol. 61, no. 5, pp. 1196-1209, 2013. Article (CrossRef Link)

[11] P. J“anis, C.-H. Yu, K. Doppler, C. Ribeiro, C. Wijting, K. Hugl, O. Tirkkonen, and V. Koivunen, "Device-to-device communication underlaying cellular communications systems," International Journal of Communications, Network and System Sciences, vol. 2, no. 3, p.169, 2009. Article (CrossRef Link)

[12] Y. Li, L. Zhang, X. Tan, and B. Cao, "An advanced spectrum allocation algorithm for the across-cell d2d communication in lte network with higher throughput," China Communications, vol. 13, no. 4, pp. 30-37, Apr. 2016. Article (CrossRef Link)

[13] G. Fodor, E. Dahlman, G. Mildh, S. Parkvall, N. Reider, G. Mikl'os, and Z. Tur'anyi, "Design aspects of network assisted device-to-device communications,” IEEE Communications Magazine, vol. 50, no. 3, pp. 170-177, Mar. 2012. Article (CrossRef Link)

[14] G. Zhang, K. Yang, P. Liu, and Y. Du, "Using full duplex relaying in device-to-device (d2d) based wireless multicast services: a two-user case,” Science China Information Sciences, vol. 58, no. 8, pp. 1-7, Aug.2015. Article (CrossRef Link)

[15] R. Ferrus, O. Sallent, G. Baldini, and L. Goratti, "Lte: the technology driver for future public safety communications," IEEE Communications Magazine, vol. 51, no. 10, pp. 154-161, Oct. 2013. Article (CrossRef Link)

[16] Z. Zhou, M. Dong, K. Ota, J. Wu, and T. Sato, "Energy efficiency and spectral efficiency tradeoff in device-to-device (d2d) communications,” IEEE Wireless Communications Letters, vol. 3, no. 5, pp. 485-488, Oct. 2014. Article (CrossRef Link) 
[17] G. Zhang, P. Liu, K. Yang, Y. Du, and Y. Hu, “Orthogonal resource sharing scheme for device-to-device communication overlaying cellular networks: a cooperative relay based approach,” Science China Information Sciences, vol. 58, no. 10, pp. 1-9, Oct. 2015.

Article (CrossRef Link)

[18] Y. Zhou, H. Liu, Z. Pan, L. Tian, J. Shi,"Energy Efficient Two-stage Cooperative Multicast Based on Device to Device Transmissions: Effect of User Density," IEEE Trans. Vehicular Technology, vol. 65, issue 9, pp. 7297-7307, Sept. 2016. Article (CrossRef Link)

[19] Y. Zhou, H. Liu, Z. Pan, L. Tian, J. Shi, “Cooperative Multicast with Location Aware Distributed Mobile Relay Selection: Performance Analysis and Optimized Design,” IEEE Trans. Vehicular Technology, Early Access Articles, Vol: 66, no: 9, pp.8291-8302 Sept. 2017. Article (CrossRef Link)

[20] X. Cheng; L. Yang; X. Shen, ”D2D for Intelligent Transportation Systems: A Feasibility Study," IEEE Transactions on Intelligent Transportation Systems, vol. 16, no. 4, pp. 1784-1793. Aug. 2015. Article (CrossRef Link)

[21] R. Zhang; X. Cheng; L. Yang; B. Jiao, "Interference Graph Based Resource Allocation (InGRA) for D2D Communications Underlaying Cellular Networks,” IEEE Transactions on Vehicular Technology, vol. 64, no. 8, pp. 3844-3850, Aug. 2015. Article (CrossRef Link)

[22] R. Zhang, X. Cheng, L. Yang, “Cooperation via Spectrum Sharing for Physical Layer Security in Device-to-Device Communications Underlaying Cellular Networks,” IEEE Transactions on Wireless Communications, vol. 15, no. 8, pp. 5651-5663, Aug. 2016. Article (CrossRef Link)

[23] J. Sun, T. Liu, X.Wang, C. Xing, H. Xiao, A. V. Vasilakos, and Z. Zhang, "Optimal Mode Selection with Uplink Data Rate Maximization for D2DAided Underlaying Cellular Networks,” IEEE Access, vol. 4, pp. 8844-8856, Nov. 2016. Article (CrossRef Link)

[24] C.-H. Yu, O. Tirkkonen, K. Doppler, and C. Ribeiro, "Power optimization of device-to-device communication underlaying cellular communication," in Proc. of 2009 IEEE International Conference on Communications.IEEE, pp. 1-5, June. 2009. Article (CrossRef Link)

[25] G. Fodor and N. Reider, "A distributed power control scheme for cellular network assisted d2d communications,” in Proc. of Global Telecommunications Conference (GLOBECOM 2011), 2011 IEEE. IEEE, pp. 1-6, Dec. 2011. Article (CrossRef Link)

[26] P. Janis, V. Koivunen, C. Ribeiro, J. Korhonen, K. Doppler, and K. Hugl, "Interference-aware resource allocation for device-to-device radio underlaying cellular networks,” in Proc. of Vehicular Technology Conference, 2009 VTC Spring 2009. IEEE 69th. IEEE, 2009, pp. 1-5. Article (CrossRef Link)

[27] Y. Jiang, Q. Liu, F. Zheng, X. Gao, and X. You, "Energy efficient joint resource allocation and power control for d2d communications,” vol. 65, no. 8, pp. 6119-6127, Aug. 2016. Article (CrossRef Link)

[28] G. Yu, L. Xu, D. Feng, R. Yin, G. Y. Li, and Y. Jiang, "Joint mode selection and resource allocation for device-to-device communications,” IEEE Transactions on Communications, vol. 62, no. 11, pp. 3814-3824, Nov. 2014. Article (CrossRef Link)

[29] H. Min, J. Lee, S. Park, and D. Hong, "Capacity enhancement using an interference limited area for device-to-device uplink underlaying cellular networks," IEEE Transactions on Wireless Communications, vol. 10, no. 12, pp. 3995-4000, Dec.2011. Article (CrossRef Link)

[30] Series M. Guidelines for evaluation of radio interface technologies for IMT-Advanced[J]. Report ITU, 2009 (2135-1). Article (CrossRef Link)

[31] Meinilä J, Kyösti P. D5. 3: WINNER+ Final Channel Models V1. 0, Jun. 2010[J]. Article (CrossRef Link)

[32] Bultitude Y J, Rautiainen T. IST-4-027756 WINNER II D1. 1.2 V1. 2 WINNER II Channel Models[J]. 2007. Article (CrossRef Link) 


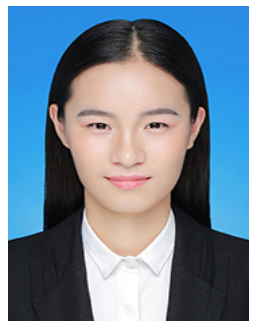

Xianxian Wang was born in 1993. She received the B.S. degree in electronic and information engineering from the Tianjin University of Science and Technology, Tianjin, China, in 2015. She is currently pursuing the M.S. degree with the Technology Research Center for Convergence Networks and Ubiquitous Services, University of Science and Technology Beijing. Her current research interests include wireless communications theory, information theory, and device-to-device communication.

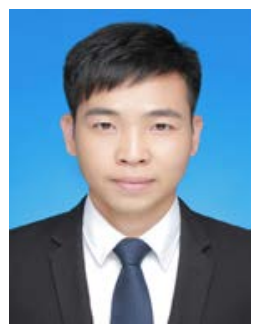

ShaoBo Lv was born in 1990. He received his B.S. degree in communication engineering from the University of Science and Technology Beijing (USTB), Beijing, China, in 2015. He is currently working toward an M.S. degree with the Technology Research Center for Convergence Networks and Ubiquitous Services, University of Science and Technology Beijing (USTB). His current research interests include wireless communications theory, information theory, and massive MIMO.

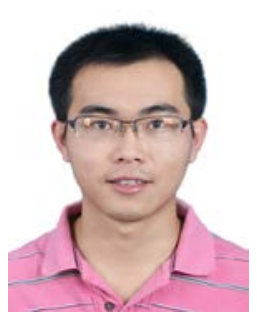

Xing Wang was born in 1984. He received his B.S. degree in Electronic Information Engineering from the Electronic Engineering Institute (EEI), Hefei, China, in 2007. After six years' work associated with astronautics, he is currently working toward an M.S degree at the Technology Research Center for Convergence Networks and Ubiquitous Services, University of Science and Technology Beijing (USTB). His current research interests include mobile wireless communications theory, deep space communication, and cooperative relay communication.

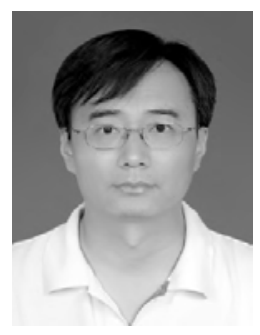

Zhongshan Zhang (SM'15) received the B.E. and M.S. degrees in computer science from the Beijing University of Post and Telecommunications (BUPT) in 1998 and 2001, respectively, and the Ph.D. degree in electrical engineering from BUPT in 2004. In 2004, he joined the DoCoMo Beijing Laboratories as an Associate Researcher, and was promoted to be a Researcher in 2005. In 2006, he joined the University of Alberta, Edmonton, AB, Canada, as a Post-Doctoral Fellow.In 2009, he joined the Department of Research and Innovation, Alcatel-Lucent, Shanghai, as a Research Scientist. From 2010 to 2011, he was with the NEC China Laboratories, as a Senior Researcher. He served or serving as a Guest Editor and/or an Editor for several technical journals, such as the IEEE Communications Magazine and the KSII Transactions on Internet and Information Systems. He is currently a Professor with the School of Computer and Communication Engineering, University of Science and Technology Beijing. His main research interests include statistical signal processing, self-organized networking, cognitive radio, and cooperative communications. 